\title{
COSMIC GAMMA-RAY BACKGROUND FROM STAR-FORMING GALAXIES
}

\author{
Brian D. Fields ${ }^{1}$, Vasiliki Pavlidoud ${ }^{2,4}$, and Tijana Prodanović ${ }^{3}$ \\ ${ }^{1}$ Departments of Astronomy and of Physics, University of Illinois, Urbana, IL 61801, USA; bdfields@illinois.edu \\ 2 California Institute of Technology, Pasadena, CA 91125, USA \\ ${ }^{3}$ Department of Physics, University of Novi Sad, Trg Dositeja Obradovića 4, 21000 Novi Sad, Serbia \\ Received 2010 May 17; accepted 2010 September 16; published 2010 October 1
}

\begin{abstract}
The origin of the extragalactic gamma-ray background is a pressing cosmological mystery. The Fermi GammaRay Space Telescope has recently measured the intensity and spectrum of this background; both are substantially different from previous measurements. We present a novel calculation of the gamma-ray background from normal star-forming galaxies. Contrary to long-standing expectations, we find that numerous but individually faint normal galaxies may comprise the bulk of the Fermi signal, rather than rare but intrinsically bright active galaxies. This result has wide-ranging implications, including: the possibility to probe the cosmic star formation history with gamma rays; the ability to infer the cosmological evolution of cosmic rays and galactic magnetic fields; and an increased likelihood of identifying subdominant components from rare sources (e.g., dark matter clumps) through their large anisotropy.
\end{abstract}

Key words: cosmic rays - galaxies: star formation - gamma rays: diffuse background - gamma rays: galaxies

Online-only material: color figure

\section{INTRODUCTION}

The Fermi Gamma-Ray Space Telescope has unveiled the high-energy cosmos with unprecedented clarity and depth. The gamma-ray sky has been known (e.g., Hunter et al. 1997; Sreekumar et al. 1998) to be dominated by diffuse emission from the Galactic plane, while at high Galactic latitudes a diffuse extragalactic gamma-ray background (EGB) has an important, and at some energies dominant, contribution. However, before Fermi, the processes dominating the diffuse emission from the Galaxy, especially above $1 \mathrm{GeV}$, were unclear and highly debated-cf. discussion on the $\mathrm{GeV}$ excess reported by the Energetic Gamma-ray Experiment Telescope (EGRET; e.g., Strong et al. 2000, and references therein). Fermi has clarified (Abdo et al. 2009a) that the dominant emission mechanism is cosmic-ray interactions with interstellar gas, which leads to gamma rays mostly from pion decay in flight, i.e., $p_{\mathrm{cr}}+p_{\text {ism }} \rightarrow$ $p p \pi^{0}$ then $\pi^{0} \rightarrow \gamma \gamma$ (Stecker 1971).

Moreover, the Fermi EGB differs from previous EGRET estimates: the intensity is fainter and the spectrum is steeper, consistent with a power law of spectral index $2.41 \pm 0.05$ and integrated intensity $I(>100 \mathrm{MeV})=(1.03 \pm 0.17) \times 10^{-5} \mathrm{~cm}^{-2} \mathrm{~s}^{-1} \mathrm{sr}^{-1}$ (Abdo et al. 2010b). Our theoretical understanding of the EGB must therefore be substantially revised in light of the new and smaller Fermi signal.

Pioneering studies investigating the origin of the EGB first considered the collective emission form star-forming galaxies (like the Milky Way), but found this to give a small EGB signal (Strong et al. 1976; Bignami et al. 1979). Pavlidou \& Fields (2002) first incorporated observations of the cosmic star formation rate (SFR), while Prodanović \& Fields (2006) made the first estimates of the pionic contribution from star-forming galaxies. In both cases, the predicted intensity was below the then-measured EGB. Blazars, the brightest extragalactic sources, have been the favored candidates (Stecker \& Salamon 1996). However, subsequent estimates of their contribution to the EGB (e.g., Mücke \& Pohl 2000; Chiang \& Mukherjee 1998;

\footnotetext{
4 Einstein Fellow.
}

Narumoto \& Totani 2007; Dermer 2007) have consistently fallen short. Fermi point-source observations suggest that unresolved blazars contribute at most $\sim 23 \%$ of the EGB; and thus the mystery has become acute (Abdo et al. 2010d).

Here, we present a more realistic model for the EGB from star-forming galaxies, constructed to use as much as possible of our substantially improved multi-wavelength observational understanding of these sources and isolating the signal from normal star formation (as opposed to starburst galaxies).

\section{FORMALISM}

The EGB intensity is an integral of the gamma-ray luminosity density $\mathcal{L}_{\gamma}$ (emissivity) over the line of sight to the cosmic horizon

$$
\frac{d I}{d E}=\frac{c}{4 \pi} \int \mathcal{L}_{\gamma}[(1+z) E, z](1+z)\left|\frac{d t}{d z}\right| d z,
$$

where $|d t / d z|=(1+z)^{-1} H(z)^{-1}=H_{0}^{-1}(1+z)^{-1}\left[(1+z)^{3} \Omega_{\mathrm{M}}+\right.$ $\left.\Omega_{\Lambda}\right]^{-1 / 2}$, with $H(z)$ the Hubble function. We adopt a $\Lambda$ CDM cosmology, with a Hubble parameter $H_{0}=71 \mathrm{~km} \mathrm{~s}^{-1} \mathrm{Mpc}^{-1}$, and cosmological constant and matter density parameters $\Omega_{\mathrm{M}}=$ 0.3 and $\Omega_{\Lambda}=0.7$, respectively.

The cosmological inputs to Equation (1) are well determined, so the EGB entirely hinges on the luminosity density $\mathcal{L}_{\gamma}$ and its connection to cosmic star formation. In this study, we construct, for the first time, a gamma-ray luminosity function (distribution of sources by gamma-ray luminosity as a function of redshift) for normal galaxies. The luminosity function is used to obtain the EGB intensity, by integrating over luminosities and redshift along photon paths.

The star-forming luminosity density due to pionic emission follows from the gamma-ray luminosity per star-forming galaxy $L_{\gamma}$ :

$$
\mathcal{L}_{\gamma}=\left\langle L_{\gamma} n_{\gamma}\right\rangle \equiv \int_{0}^{L_{\max }} L_{\gamma} n_{\gamma}\left(L_{\gamma}\right) d L_{\gamma},
$$

where $n_{\gamma}$ is the comoving number density of gamma-rayluminous galaxies. The average is taken over the distribution 
of star-forming galaxy properties at redshift $z$, i.e., the gammaray luminosity function which gives the comoving number $n_{\gamma}\left(L_{\gamma}\right) d L_{\gamma}$ of star-forming galaxies with luminosity in the range $\left(L_{\gamma}, L_{\gamma}+d L_{\gamma}\right)$; out to a maximum $L_{\max }$ determined below.

A galaxy's pionic gamma-ray flux scales with the cosmic-ray flux (projectiles) and the amount of interstellar gas (targets) in the galaxy. Specifically, a galaxy's rest-frame pionic gamma-ray luminosity (photon counts per unit time) is given by

$$
\begin{aligned}
L_{\gamma}\left(E_{\mathrm{em}}\right) & =\int \Gamma_{\pi^{0} \rightarrow \gamma \gamma}\left(E_{\mathrm{em}}\right) n_{\mathrm{H}} d V_{\mathrm{ism}} \\
& =\Gamma_{\pi^{0} \rightarrow \gamma \gamma}\left(E_{\mathrm{em}}\right) \mathcal{N}_{p} \propto \Phi_{p} M_{\mathrm{gas}}
\end{aligned}
$$

with $E_{\mathrm{em}}$ the photon energy in the emitting frame. Here, the pionic gamma-ray production rate per interstellar $\mathrm{H}$ atom is $\Gamma_{\pi^{0} \rightarrow \gamma \gamma}\left(E_{\mathrm{em}}\right)=\left\langle\Phi_{p} d \sigma_{\pi^{0} \rightarrow \gamma \gamma} / d E_{\mathrm{em}}\right\rangle \propto \Phi_{p}$ and is proportional to the galaxy's volume-averaged cosmic-ray proton flux $\Phi_{p}$ (Pohl 1994; Persic \& Rephaeli 2010); the cross-section $d \sigma_{\pi^{0} \rightarrow \gamma \gamma} / d E_{\mathrm{em}}$ is understood to include effects of pion multiplicity and of helium and heavier elements in the cosmic rays and interstellar medium. The factor $\mathcal{N}_{p}=\int n_{\mathrm{H}} d V_{\text {ism }}$ gives the number of hydrogen atoms in the galaxy's interstellar medium, summed over all states-molecular, atomic, and ionized. This term is fixed by the galaxy's total interstellar gas mass via $\mathcal{N}_{\mathrm{H}}=X_{\mathrm{H}} M_{\text {gas }} / m_{p}$, where $X_{\mathrm{H}} \approx 0.70$ is the hydrogen mass fraction.

Since cosmic rays are thought to be predominantly accelerated in sites associated with massive star formation (supernova remnants, massive stellar winds, pulsars; see, e.g., Lacki et al. 2010, and references therein), their flux in Equation (3) should scale with the SFR in the galaxy. Observations by Fermi and TeV telescopes HESS and VERITAS have confirmed this expectation through observations of star-forming galaxies (Abdo et al. 2010a, 2010c, 2010e; Acero et al. 2009; VERITAS Collaboration et al. 2009), for which both SFR and total gas mass were independently known. Thus, the cosmic-ray flux is set by the competition of particle acceleration and losses that we assume are dominated by escape, as they are in the Milky Way. These selfregulating processes tend toward an equilibrium $\Phi_{p} \propto \Lambda_{\mathrm{esc}} \psi$, where $\psi$ is the SFR and $\Lambda_{\text {esc }}$ is the escape path length (assumed constant). We thus adopt the scaling,

$$
L_{\gamma} \propto M_{\text {gas }} \psi
$$

of gamma-ray luminosity with a galaxy's gas mass and SFR $\psi$ (Pavlidou \& Fields 2001).

The scaling in Equation (5) represents a central ansatz regarding gamma-ray production in Milky-Way-like galaxies whose cosmic-ray losses are dominated by escape. This physically motivated relation now has support from Fermi observation of star-forming galaxies for which the product $M_{\text {gas }} \psi$ ranges over several orders of magnitude. A second ansatz in our model is our adoption of a universal cosmic-ray spectrum of index $s_{\mathrm{cr}}=2.75$. At energies away from the peak the gamma-ray spectrum has the same index: $s_{\gamma, \text { had }}=s_{\mathrm{cr}}$. Even though both approximations are certainly simplifying, they represent important benchmark cases, against which more sophisticated models can be tested.

The scaling law of Equation (5) allows us to determine the gamma-ray output of any star-forming galaxy, but only once we normalize our results to a system in which the cosmic-ray and/or gamma-ray properties are known. We choose to normalize to the Milky Way, where the local cosmic-ray flux is well measured, and the global SFR is also known. We assume that the ratio of cosmic-ray flux to SFR should be constant for all normal galaxies, i.e., that

$$
\frac{\Gamma_{\pi^{0} \rightarrow \gamma \gamma}}{\Gamma_{\pi^{0} \rightarrow \gamma \gamma}^{\mathrm{MW}}}=\frac{\Phi_{\mathrm{cr}}}{\Phi_{\mathrm{cr}, \mathrm{MW}}}=\frac{R_{\mathrm{SN}}}{R_{\mathrm{SN}, \mathrm{MW}}}=\frac{\psi}{\psi_{\mathrm{MW}}} .
$$

This scaling encodes the long-standing notion that supernova remnants accelerate hadronic cosmic rays (leading to pionic emission; e.g., Abdo et al. 2009c).

We thus find that for normal galaxies,

$$
\begin{aligned}
L_{\gamma}\left(M_{\mathrm{gas}}, \psi\right) & =X_{\mathrm{H}} \Gamma_{\pi^{0} \rightarrow \gamma \gamma}^{\mathrm{MW}} \frac{M_{\mathrm{gas}}}{m_{p}} \frac{\psi}{\psi_{\mathrm{MW}}} \\
& =1.7 \times 10^{42} \mathrm{~s}^{-1}\left(\frac{M_{\mathrm{gas}}}{10^{10} M_{\odot}}\right)\left(\frac{\psi}{1 M_{\odot} \mathrm{yr}^{-1}}\right) .
\end{aligned}
$$

We adopt the photon emission per hydrogen atom derived from Fermi diffuse Galactic observations at medium latitudes for photons > $100 \mathrm{MeV}: \quad \Gamma_{\pi \rightarrow \gamma \gamma}^{\mathrm{MW}}=2.0 \times 10^{-25} \mathrm{~s}^{-1} \mathrm{H}$-atom ${ }^{-1}$ (Abdo et al. 2009b). This value represents a large-scale $(\sim \mathrm{kpc})$ spatial averaging of Galactic cosmic-ray properties, which is appropriate for our global calculation.

We can now write the gamma-ray luminosity density for normal galaxies,

$$
\mathcal{L}_{\gamma}\left(E_{\mathrm{em}}, z\right)=X_{\mathrm{H}} \frac{\Gamma_{\pi^{0} \rightarrow \gamma \gamma}^{\mathrm{MW}}\left(E_{\mathrm{em}}\right)}{\psi_{\mathrm{MW}}} \frac{\left\langle M_{\mathrm{gas}}(z)\right\rangle}{m_{p}} \dot{\rho}_{\star}(z),
$$

in terms of the cosmic SFR $\dot{\rho}_{\star}(z)=\left\langle\psi n_{\text {gal }}\right\rangle$. We define a mean interstellar gas mass as

$$
\left\langle M_{\mathrm{gas}}(z)\right\rangle \equiv \frac{\left\langle M_{\mathrm{gas}} \psi n_{\mathrm{gal}}\right\rangle}{\left\langle\psi n_{\mathrm{gal}}\right\rangle}=\frac{\left\langle M_{\mathrm{gas}} \psi n_{\mathrm{gal}}\right\rangle}{\dot{\rho}_{\star}} .
$$

The assumption that losses are escape dominated and uniform across galaxies can only be approximately valid at best. This is a major uncertainty in our model, which will benefit from future data on the EGB and resolved galaxies. For example, even the leaky-box model can generalize Equation (5) to $L_{\gamma} \propto \Lambda_{\text {esc }} \psi M_{\text {gas }}$; variations in the energy dependence of the escape length $\Lambda_{\text {esc }}$ would also change the cosmic-ray and photon spectral indices which we take as universal.

Indeed, Fermi observations of the Large Magellanic Cloud (LMC) suggest that cosmic-ray confinement and propagation are non-trivial (Abdo et al. 2010c). Moreover, starburst galaxies show very high cosmic-ray intensities within small volumes where inelastic collisions compete with, and sometimes dominate, outflows to regulate cosmic-ray losses (Paglione et al. 1996; Lacki et al. 2010; Torres et al. 2004; Thompson et al. 2007; Persic \& Rephaeli 2010; Stecker 2007). For this reason, Equation (7) provides a rough description of normal escapedominated galaxies only; we do not expect it to hold for starburst galaxies, which we will exclude below.

\subsection{Gas Mass and Star Formation Rate}

We can infer a galaxy's interstellar gas mass at a given SFR, via the well-established Kennicutt-Schmidt law (Schmidt 1959; Kennicutt 1998). The surface densities for star formation and gas are found to be correlated via $\dot{\Sigma}_{\star} / M_{\odot} \mathrm{yr}^{-1} \mathrm{kpc}^{-2}=$ $(2.5 \pm 0.7) \times 10^{-4}\left(\Sigma_{\text {gas }} / M_{\odot} \mathrm{pc}^{-2}\right)^{x}$ with $x=1.4 \pm 0.15$. Both normal and starburst galaxies follow this correlation, but normal 
galaxies populate $\dot{\Sigma}_{\star \text {,normal }} \lesssim \dot{\Sigma}_{\star \text { mormal }}^{\text {max }} \equiv 0.4 M_{\odot} \mathrm{yr}^{-1} \mathrm{kpc}^{-2}$ while starbursts occupy the opposite regime (Kennicutt 1998).

To recover a relationship between the global galactic SFR $\psi=\pi r_{\text {disk }}^{2} \dot{\Sigma}_{\star}$ and gas mass $M_{\text {gas }}=\pi r_{\text {disk }}^{2} \Sigma_{\text {gas }}$ requires a galactic disks scale length $r_{\text {disk }}$. We take $r_{\text {disk }}=18.9 \mathrm{kpc} /(1+z)$; observations (Erb et al. 2006) indicate that this choice is uncorrelated with galaxy mass and SFR at a fixed $z$. Combining this with the Kennicutt-Schmidt law, we find

$$
M_{\text {gas }}(\psi, z)=2.8 \times 10^{9} M_{\odot}(1+z)^{-\beta}\left(\frac{\psi}{1 M_{\odot} \mathrm{yr}^{-1}}\right)^{\omega},
$$

where $\beta=2(1-1 / x)=0.571$ and $\omega=1 / x=0.714$. In our model, normal galaxies extend to

$$
\psi \leqslant \psi_{\text {normal }}^{\max }(z) \equiv \pi r_{\text {disk }}(z)^{2} \Sigma_{\star, \text { normal }}^{\max } \simeq \frac{450}{(1+z)^{2}} M_{\odot} \mathrm{yr}^{-1},
$$

this cutoff becomes important for $z \gtrsim 1$.

Combining Equations (10) and (7), we can express a galaxy's gamma-ray luminosity in terms of its SFR and redshift:

$$
L_{\gamma}(\psi, z) \propto(1+z)^{-\beta}\left(\frac{\psi}{1 M_{\odot} \mathrm{yr}^{-1}}\right)^{\omega+1}
$$

Available Fermi data on resolved $z \approx 0$ star-forming galaxies are consistent with this scaling (Abdo et al. 2010a).

\subsection{Gamma-ray Luminosity Function}

A galaxy's luminosity in the $\mathrm{H} \alpha$ line provides a wellestablished tracer of SFR: $L_{\mathrm{H} \alpha} / 1.26 \times 10^{34} \mathrm{~W}=\psi / 1 M_{\odot} \mathrm{yr}^{-1}$ (Hopkins 2004). The $\mathrm{H} \alpha$ distribution of galaxies (luminosity function) is related to the cosmic SFR density via $\dot{\rho}_{\star}(z)=$ $\langle\psi n\rangle \propto \int L_{\mathrm{H} \alpha} n\left(L_{\mathrm{H} \alpha}, z\right) d L_{\mathrm{H} \alpha}$, which by now is well measured as a function of redshift both by $\mathrm{H} \alpha$ and by other methods, so that its shape is well determined observationally.

The gamma-ray luminosity density is a different moment of the $\mathrm{H} \alpha$ luminosity function $\mathcal{L}_{\gamma}(z)=\langle\psi n\rangle \propto(1+$ $z)^{-\beta} \int_{\mathrm{H} \alpha}^{L_{\max }} L_{\mathrm{H} \alpha}^{\omega} n\left(L_{\mathrm{H} \alpha}, z\right) d L_{\mathrm{H} \alpha}$ via our scaling laws above. We see that the gamma-ray luminosity distribution (i.e., luminosity function) at a given redshift follows directly from the distribution of SFRs, as traced by $\mathrm{H} \alpha$. Since we consider here only normal galaxies, we include only galaxies with $\psi \leqslant \psi_{\text {normal }}^{\text {max }}$ (Equation (11)) which sets corresponding limits $L_{\mathrm{H} \alpha} \leqslant L_{\mathrm{H} \alpha}^{\max }$ and $L_{\gamma} \leqslant L_{\gamma}^{\max }$ in Equation (2).

Current data on the $\mathrm{H} \alpha$ luminosity function can be fit to a Schechter function, of the form $n(L, z) d L=$ $n_{*}\left(L / L_{*}\right)^{-\alpha} \exp \left(-L / L_{*}\right) d L / L_{*}$, where $L=L_{\mathrm{H} \alpha}$. Present data are consistent with the value $\alpha=1.43$, found for $z=0$, persisting for all redshifts. Data also fix the $z=0$ values, $n_{*}(0)=1.0 \times 10^{-3} \mathrm{Mpc}^{-3}$ and $L_{*}(0)=9.5 \times 10^{34} \mathrm{~W}$, for $h=0.71$ (Nakamura et al. 2004). This corresponds to a SFR $\psi\left(L_{*}\right)=7.5 M_{\odot} \mathrm{yr}^{-1}$.

However, observations currently do not give unambiguous solutions for the other two parameters, the characteristic comoving density of star-forming galaxies $n_{*}$ and the characteristic $\mathrm{H} \alpha \mathrm{lu}$ minosity $L_{*}$ (Hopkins 2004). Moreover, it is unclear whether and how each parameter evolves with redshift.

Two limiting cases bracket the possible behaviors of the $\mathrm{H} \alpha$ luminosity function and thus of cosmic star formation. In the case of pure luminosity evolution, the comoving density of stars is fixed, $n_{*}=$ const independent of $z$, and all redshift evolution lies in $L_{*}=L_{*}(z)$. Conversely, pure density evolution places the redshift evolution in the density scale $n_{*}(z)$ while setting $L_{*}=$ const. In each of these two limits, we can find the redshift dependence of the parameters via the requirement $\langle L n\rangle \propto \dot{\rho}_{\star}$. The redshift history of cosmic-star formation is well known (Horiuchi et al. 2009), and we encode this in the dimensionless "shape" function

$$
S(z) \equiv \dot{\rho}_{\star}(z) / \dot{\rho}_{\star}(0) .
$$

We then have $L_{*}(z) / L_{*}(0)=S(z)$ in the case of pure luminosity evolution and $n_{*}(z) / n_{*}(0)=S(z)$ in the case of pure density evolution.

At a given redshift, Equation (9) gives the scaling $\left\langle M_{\text {gas }}\right\rangle=$ $\left\langle M_{\text {gas }} \psi n\right\rangle / \dot{\rho}_{\star} \propto\left\langle M_{\text {gas }} \psi n\right\rangle / S(z)$. For our $\mathrm{H} \alpha$ luminosity function and Kennicutt-Schmidt relation, we find a local value of $\left\langle M_{\text {gas }}\right\rangle_{z=0}=6.8 \times 10^{9} M_{\odot}$. At other redshifts, we have $\left\langle M_{\text {gas }} \psi n\right\rangle \propto(1+z)^{-\beta} L_{*}(z)^{\omega} S(z)$, and so $\left\langle M_{\text {gas }}\right\rangle \propto(1+$ $z)^{-\beta} L_{*}(z)^{\omega}$. Thus, for the pure luminosity case $L_{*} \propto S(z)$, we find that the gas mass strongly evolves as $\left\langle M_{\text {gas }}\right\rangle \propto$ $(1+z)^{-\beta} S(z)^{\omega}$, in response to the strongly changing SFR. Consequently, the factor of 10 rises in cosmic star formation at $z \simeq 1$ implies a net gamma-ray luminosity increase of a factor $\simeq 30$. On the other hand, in the pure density evolution case, galaxy SFRs are constant, $L_{*}(z)=$ const, so that the mean gas mass $\left\langle M_{\text {gas }}\right\rangle \propto(1+z)^{-\beta}$ actually decreases with redshift, while the comoving number of star-forming galaxies increases, but the net enhancement at high redshift is smaller than in the pure luminosity evolution case. This key difference leads to the factor $\sim 4$ between the EGB predictions for the pure density and pure luminosity evolution cases seen in Figure 1.

\section{RESULTS AND CONCLUSIONS}

Our full numerical calculation uses a Milky Way pionic source spectrum whose shape is derived from Pfrommer \& Enßlin (2004), calibrated to observations by normalizing the $>100 \mathrm{MeV}$ photon emission per hydrogen atom to the Fermi result at intermediate Galactic latitudes (Abdo et al. 2009b). The cosmic SFR is from Horiuchi et al. (2009). For the Milky Way SFR, used to normalize the cosmic-ray flux/SFR ratio, we use the recent estimate of Robitaille \& Whitney (2010; $\psi_{\mathrm{MW}}=1 M_{\odot} \mathrm{yr}^{-1}$, a factor of three lower than earlier work).

Figure 1 shows our results for the normal-galaxy contribution to the EGB. We plot predictions for the limiting cases of pure luminosity and of pure density evolution. The uncertainties in the model inputs, summed in quadrature, propagate into the displayed error band that applies to each curve, which we estimate to be a factor of $10^{ \pm 0.3}$, resulting from uncertainties of: $30 \%$ in pionic emissivity (Abdo et al. 2009b), $40 \%$ in the normalization of the Galactic SFR (Robitaille \& Whitney 2010), $40 \%$ in the cosmic SFRs (Horiuchi et al. 2009), and $25 \%$ in the luminosity scaling in Equation (9). The true systematic uncertainty would also reflect the idealizations in our model (universal cosmic-ray spectra and confinement). These errors are hard to estimate but in any case imply that the uncertainty range in Figure 1 is a lower bound to the error budget.

Within errors, our predictions for both limiting models fall at or below the level of the Fermi data, where the data seem to support the pure luminosity evolution case that explains nearly the entire signal. Comparing central values, this model gives $\approx 50 \%$ of the Fermi EGB $\lesssim 10 \mathrm{GeV}$. Thus, unresolved normal galaxies make a substantial and likely dominant contribution to the observed EGB, without overpredicting the signal. Even 


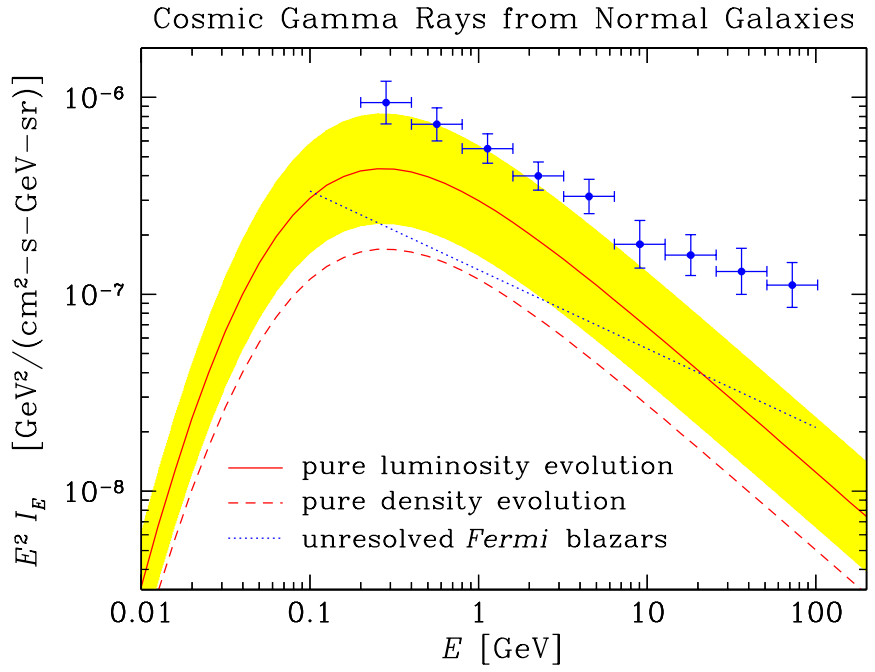

Figure 1. Normal-galaxy contribution to the EGB. Curves represent two limiting cases of cosmic star formation: pure luminosity and pure density evolution. Colored error band illustrates the factor $10^{ \pm 0.3}$ uncertainty in the normalization of both theory curves. Fermi data are from Abdo et al. (2010b). Dotted line: unresolved blazar EGB upper limit (Abdo et al. 2010d).

(A color version of this figure is available in the online journal.)

the pure density evolution case accounts for a minimum of $20 \%$ of the EGB around $0.3 \mathrm{GeV}$; this provides a lower limit to the normal-galaxy signal. Thus, any other EGB sources (Stecker \& Salamon 1996; Dermer 2007; Abdo et al. 2010d; Faucher-Giguère \& Loeb 2010) must contribute no more than the remaining $80 \%$ of the data. Indeed, the LAT team upper limit to the blazar EGB contribution shown in Figure 1 is comparable to our lower limit (Abdo et al. 2010d).

The spectral shapes of the two limiting cases are very similar: the peak in $E^{2} d I / d E$ lies at $\sim 0.3 \mathrm{GeV}$ because the bulk of the signal comes from $z \sim 1$. These models predict that the EGB turns over for $E \lesssim 0.3 \mathrm{GeV}$, a testable prediction of our model. For hadronic emission, the high-energy spectral index is the same as the underlying proton spectral index, here $s_{\gamma, \text { had }}=s_{\text {cr }}=2.75$; this is somewhat steeper than the Fermi single-power-law fit $s_{\text {obs }}=2.41 \pm 0.05$. Consequently, our predictions at high energies $(\gtrsim 10 \mathrm{GeV})$ fall below the data. If normal galaxies had a distribution of cosmic-ray spectral indices, the resulting EGB spectrum would steepen at high energies where the hardest sources would dominate, developing a convex tail. Indeed, the Fermi EGB data suggest a slight flattening of slope around $E \gtrsim 10 \mathrm{GeV}$, which might hint at such transition.

A galaxy with characteristic $\mathrm{H} \alpha$ luminosity $L_{*}$ has $L_{\gamma}^{*}(>100 \mathrm{MeV})=1.4 \times 10^{43} \mathrm{~s}^{-1}$. Such objects have flux $F$ if they lie at distances $r_{*}=\left(L_{\gamma}^{*} / 4 \pi F\right)^{1 / 2}=$ $11 \mathrm{Mpc}\left(10^{-9} \mathrm{~cm}^{-2} \mathrm{~s}^{-1} / F\right)^{1 / 2}$. Thus, Fermi should eventually resolve

$$
N(>F) \sim 4 \pi r_{*}^{3} n_{*}(0) / 3=5\left(\frac{10^{-9} \mathrm{~cm}^{-2} \mathrm{~s}^{-1}}{F}\right)^{3 / 2}
$$

normal galaxies, consistent with 2-3 detections to date (the LMC, Small Magellanic Cloud, and perhaps M31; Abdo et al. 2010c; The Fermi/LAT collaboration \& Abdo 2010; Ögelman et al. 2010).

Our results do not account for starburst galaxies, nor for inverse Compton emission from any star-forming galaxies; these must contribute to the star-forming EGB and could have hard spectra dominating $\gtrsim 10 \mathrm{GeV}$. We have also neglected gammaray attenuation by extragalactic background light (important at $E \gtrsim 30 \mathrm{GeV}$; e.g., Stecker et al. 2006, and references therein). We will address these issues in future work.

The amplitude and configuration of magnetic fields in a galaxy have an additional effect on the scaling of cosmic-ray flux with SFR. Confirmation that normal galaxies comprise the bulk of the Fermi signal would constitute a unique probe of the evolution of these magnetic fields between the redshift of peak star formation and today.

Because of their ubiquity, normal galaxies produce the smallest anisotropies in the EGB, far less than blazars or other proposed sources. Thus, by studying the observed EGB anisotropy as a function of energy, it may be possible to disentangle the spectrum and amplitude of the normal-galaxy contribution from that of other sources (Ando \& Pavlidou 2009). Moreover, because normal galaxies seem to dominate the Fermi EGB, their small contribution to anisotropies will fortuitously provide the optimal chance of finding smaller and more exotic sources in the observed signal (Siegal-Gaskins \& Pavlidou 2009).

It is a pleasure to thank Marco Ajello, Chuck Dermer, Hai Fu, Troy Porter, and Andy Strong for stimulating discussions. B.D.F. thanks the Goddard Space Flight Center for hospitality while some of this work was done. This work was partially supported by NASA, via Fermi GI Program grants NNX09AT74G and NNX09AU01G, and the Astrophysics Theory Program through award NNX10AC86G. V.P. acknowledges NASA support through Einstein Postdoctoral Fellowship grant number PF8-90060 awarded by the Chandra X-ray Center, which is operated by the Smithsonian Astrophysical Observatory for NASA under contract NAS8-03060. V.P. thanks the Physics Department at the University of Crete for their hospitality during part of this work. The work of T.P. is supported in part by the Provincial Secretariat for Science and Technological Development, and by the Ministry of Science of the Republic of Serbia under project 141002B.

\section{REFERENCES}

Abdo, A. A., et al. 2009a, Phys. Rev. Lett., 103, 251101

Abdo, A. A., et al. 2009b, ApJ, 703, 1249

Abdo, A. A., et al. 2009c, ApJ, 706, L1

Abdo, A. A., et al. 2010a, ApJ, 709, L152

Abdo, A. A., et al. 2010b, Phys. Rev. Lett., 104, 101101

Abdo, A. A., et al. 2010c, A\&A, 512, A7

Abdo, A. A., et al. 2010d, ApJ, 720, 435

Abdo, A. A., et al. 2010e, ApJS, 188, 405

Acero, F., et al. 2009, Science, 326, 1080

Ando, S., \& Pavlidou, V. 2009, MNRAS, 400, 2122

Bignami, G. F., Fichtel, C. E., Hartman, R. C., \& Thompson, D. J. 1979, ApJ, 232, 649

Chiang, J., \& Mukherjee, R. 1998, ApJ, 496, 752

Dermer, C. D. 2007, in AIP Conf. Proc. 921, The First GLAST Symposium, ed. S. Ritz, P. Michelson, \& C. A. Meegan (Melville, NY: AIP), 122

Erb, D. K., Steidel, C. C., Shapley, A. E., Pettini, M., Reddy, N. A., \& Adelberger, K. L. 2006, ApJ, 646, 107

Faucher-Giguère, C.-A., \& Loeb, A. 2010, J. Cosmol. Astropart. Phys., 1, 5 Hopkins, A. M. 2004, ApJ, 615, 209

Horiuchi, S., Beacom, J. F., \& Dwek, E. 2009, Phys. Rev. D, 79, 083013

Hunter, S. D., et al. 1997, ApJ, 481, 205

Kennicutt, R. C., Jr. 1998, ApJ, 498, 541

Lacki, B. C., Thompson, T. A., Quataert, E., Loeb, A., \& Waxman, E. 2010, arXiv: 1003.3257

Mücke, A., \& Pohl, M. 2000, MNRAS, 312, 177

Nakamura, O., Fukugita, M., Brinkmann, J., \& Schneider, D. P. 2004, AJ, 127, 2511 
Narumoto, T., \& Totani, T. 2007, Ap\&SS, 309, 73

Ögelman, H., Aksaker, N., Anilan, S., Dereli, H., Emrahoğlu, N., \& Yegingil, I. 2010, arXiv:1008.2537

Paglione, T. A. D., Marscher, A. P., Jackson, J. M., \& Bertsch, D. L. 1996, ApJ, 460, 295

Pavlidou, V., \& Fields, B. D. 2001, ApJ, 558, 63

Pavlidou, V., \& Fields, B. D. 2002, ApJ, 575, L5

Persic, M., \& Rephaeli, Y. 2010, MNRAS, 403, 1569

Pfrommer, C., \& Enßlin, T. A. 2004, A\&A, 413, 17

Pohl, M. 1994, A\&A, 287, 453

Prodanović, T., \& Fields, B. D. 2006, ApJ, 645, L125

Robitaille, T. P., \& Whitney, B. A. 2010, ApJ, 710, L11

Schmidt, M. 1959, ApJ, 129, 243

Siegal-Gaskins, J. M., \& Pavlidou, V. 2009, Phys. Rev. Lett., 102, 241301
Sreekumar, P., et al. 1998, ApJ, 494, 523

Stecker, F. W. 1971, Cosmic Gammay Rays (NASA SP 249; Washington, DC: NASA)

Stecker, F. W. 2007, Astropart. Phys., 26, 398

Stecker, F. W., Malkan, M. A., \& Scully, S. T. 2006, ApJ, 648, 774

Stecker, F. W., \& Salamon, M. H. 1996, ApJ, 464, 600

Strong, A. W., Moskalenko, I. V., \& Reimer, O. 2000, ApJ, 537, 763

Strong, A. W., Wolfendale, A. W., \& Worrall, D. M. 1976, MNRAS, 175, $23 \mathrm{P}$

The Fermi/LAT collaboration \& Abdo, A. A. 2010, arXiv:1008.2127

Thompson, T. A., Quataert, E., \& Waxman, E. 2007, ApJ, 654, 219

Torres, D. F., Reimer, O., Domingo-Santamaría, E., \& Digel, S. W. 2004, ApJ, 607, L99

VERITAS Collaboration, et al. 2009, Nature, 462, 770 